\title{
Diseño, elaboración y validación de un simulador realista y de bajo costo para exploración cardiaca
}

\author{
Ulises Sánchez-Vásquez, ${ }^{1 *}$ Alba B. Daniel-Guerrero, ${ }^{2}$ Eduardo Méndez-Gutiérrez, ${ }^{2}$ \\ Sara Morales-López, Isabel I. Tovar-Lozano, ${ }^{2}$ Marco A. Martínez-Rodríguez ${ }^{2}$ e Israel E. Uribe-Campos ${ }^{2}$ \\ ${ }^{1}$ Centro Médico ABC, Departamento de Anestesiología; ${ }^{2}$ Universidad Nacional Autónoma de México, Facultad de Medicina, Departamento de \\ Integración de Ciencias Médicas. Ciudad de México, México
}

\section{Resumen}

Introducción: La exploración cardiaca es una competencia clínica fundamental que requiere exposición o entrenamiento continuo. La baja disponibilidad y accesibilidad de pacientes con patología cardiaca constituye una barrera para adquirir esta competencia. Se han documentado inadecuadas habilidades de auscultación cardiaca en estudiantes de medicina, residentes y médicos graduados. Objetivo: Elaborar y validar un simulador de alta fidelidad y bajo costo para exploración cardiaca. Métodos: Se diseñó y elaboró un simulador para exploración cardiaca, realista y de bajo costo capaz de reproducir ruidos cardiacos normales. Posteriormente se realizó la validación del simulador por un grupo de expertos que emitieron su opinión de acuerdo con una escala tipo Likert. Resultados: El 94 \% afirmó que el simulador motiva el aprendizaje de la exploración cardiaca y 92 \% lo consideró un modelo realista; 91 \% consideró que el simulador es una herramienta atractiva para fortalecer el aprendizaje y 98 \% recomendó seguir utilizándolo. Conclusiones: El uso del simulador facilita la adquisición de competencias y estimula el aprendizaje en el estudiante, lo cual puede ser atribuido a la práctica deliberada, a un mayor tiempo de exposición y a la interacción cognitiva.

PALABRAS CLAVE: Simulador cardiaco. Bajo costo. Realismo. Enseñanza. Competencia.

\section{Design, assembly and validation of a low-cost, high-fidelity simulator for heart exploration}

\begin{abstract}
Introduction: Heart exploration is an essential clinical competence that requires continuous training and exposure. Low availability and accessibility to patients with heart disease constitutes a barrier to acquiring this competence. Inadequate cardiac auscultation skills in medical students, residents, and graduate physicians have been documented. Objective: To develop and validate a low-cost, high-fidelity simulator for heart exploration. Methods: A low-cost, high-fidelity heart examination simulator capable of reproducing normal cardiac sounds was designed and developed. Subsequently, the simulator was validated by a group of experts who gave their opinion according to a Likert scale. Results: Ninety-four percent agreed that the simulator motivates the learning of heart exploration, and $92 \%$ considered it to be a realistic model; $91 \%$ considered that the simulator is an attractive tool to reinforce learning and $98 \%$ recommended its further use. Conclusions: The use of the simulator facilitates the acquisition of skills and stimulates learning in the student, which can be attributed to repeated practice, longer exposure time and cognitive interaction.
\end{abstract}

KEY WORDS: Heart simulator. Low cost. Realism. Education. Competence.

Correspondencia:

*Ulises Sánchez-Vásquez

E-mail: ulises.sanchez.vasquez@gmail.com

DOI: 10.24875/GMM.20005688

0016-3813/@ 2020 Academia Nacional de Medicina de México, A.C. Publicado por Permanyer. Este es un artículo open access bajo la licencia
Fecha de recepción: 18-12-2019

Fecha de aceptación: 26-05-2020
Gac Med Mex. 2021;157:25-29

Disponible en PubMed

www.gacetamedicademexico.com CC BY-NC-ND (http://creativecommons.org/licenses/by-nc-nd/4.0/). 


\section{Introducción}

La exploración cardiaca (EC), aspecto esencial en la exploración física, es una experiencia multisensorial que requiere la integración de los datos obtenidos mediante inspección, palpación, percusión y auscultación en el contexto de síntomas iniciales y antecedentes del paciente. ${ }^{1}$ La adquisición de esta competencia es una habilidad clínica fundamental que requiere exposición continua para que sea alcanzada. ${ }^{2}$ Cuando la EC es realizada correctamente, la mayoría de las afecciones estructurales cardiacas pueden ser diagnosticadas con exactitud o como parte de un diagnóstico diferencial. ${ }^{1}$ Para volverse competente en esta habilidad, un médico debe explorar repetidamente a pacientes con una gran variedad de afecciones cardiacas, de ahí que la disponibilidad y accesibilidad de los pacientes con patología cardiaca constituye una barrera para la adquisición de esta competencia. ${ }^{3}$

Se han documentado inadecuadas habilidades de auscultación cardiaca en estudiantes de medicina y en médicos, lo que indica que se debe prestar mayor atención a la enseñanza de esta competencia. Con excepción de los cardiólogos, no existe una mejora en las habilidades de auscultación después del tercer año de medicina en residentes o en médicos graduados. ${ }^{1,4}$ Para vencer este obstáculo se han desarrollado formatos de enseñanza alternativos, como grabaciones de audio, multimedia y simuladores cardiopulmonares de alta fidelidad, por ejemplo Harvey y Simulator-K..$^{5,6}$ Se ha demostrado que una breve sesión teórica combinada con práctica en un simulador cardiopulmonar mejora la exactitud del diagnóstico en patología cardiaca simulada, así como la puntuación en exámenes teóricos y prácticos. ${ }^{7-9} \mathrm{La}$ evidencia respalda mejores prácticas para la educación basada en simulación, tales como rango de dificultad, práctica repetitiva, práctica distribuida, interacción cognitiva, estrategias de aprendizaje múltiple o individualizado, aprendizaje de dominio, mayor tiempo de exposición, variación clínica e interrogación (debriefing). ${ }^{10}$

Ante la importancia de la auscultación para la detección de anomalías estructurales cardiacas y la evidencia de falta de mejora en esta habilidad en estudiantes de medicina y en médicos graduados debido a la poca exposición a la patología cardiaca, se diseñó y elaboró un simulador clínico realista y de bajo costo para exploración cardiaca, cuya efectividad se validó con un grupo de expertos, con el objetivo de mejorar la habilidad de auscultación cardiaca en estudiantes de medicina de la Universidad Nacional Autónoma de México.

\section{Métodos}

El diseño del simulador fue realizado en el Centro de Enseñanza y Certificación de Aptitudes Médicas del Departamento de Integración de Ciencias Médicas, en la Facultad de Medicina de la Universidad Nacional Autónoma de México. La presente investigación estuvo aprobada por el Comité de Investigación del Departamento de Integración de Ciencias Médicas y no se requirió consentimiento informado.

El simulador para la exploración cardiaca recibió el nombre Hearthur y está constituido por tres elementos:

1. Armado del simulador: se tomó el torso de un maniquí, del cual la parrilla costal fue perforada de acuerdo con el nivel anatómico de los focos de auscultación cardiaca (Figura 1A). Alrededor de estas perforaciones, para el paso del sonido se realizaron cuatro orificios donde se colocaron luces LED en forma de cruz, es decir, en las partes superior, inferior, lateral derecha y lateral izquierda (Figura 1B). Posteriormente, $90 \%$ del diámetro del torso fue cubierto con hule espuma de $0.5 \mathrm{~cm}$ de grosor, color piel, ajustado con pegamento silicón; en la parte posterior se colocó una línea de $50 \mathrm{~cm}$ de adhesivo velcro que permite retirar la cubierta para su limpieza (Figura 1C). El torso fue instalado en una base de madera de $60 \times 45 \mathrm{~cm}$, previamente pintada (Figura 1D). En la parte posterior se construyó una caja de madera de $15 \times 8 \times 4 \mathrm{~cm}$ que contiene una batería de $9 \mathrm{~V}$, una perilla de encendido/apagado y una entrada de audio universal (Figura 1E).

2. Circuito eléctrico y de audio: fue colocado en la pared interna de la parrilla costal. El circuito constó de cinco bocinas y alrededor de cada bocina, cuatro focos LED rojos de $5 \mathrm{~mm}$ con resistencia de carbón de $1 / 2$ vatio a $5 \%$ de $330 \Omega$, unidas con pasta para soldadura a un cable tipo micrófono doble de 22 AWG que va a un broche porta batería de $9 \mathrm{~V}$. La perilla de dos posiciones (on-off) tiene un switch de balancín, de un polo y un tiro, con piloto de 12 Vcc (Figura 2).

3. Ruidos cardiacos: mediante un estetoscopio convencional y un micrófono de alta resolución se grabaron los ruidos cardiacos de un hombre 

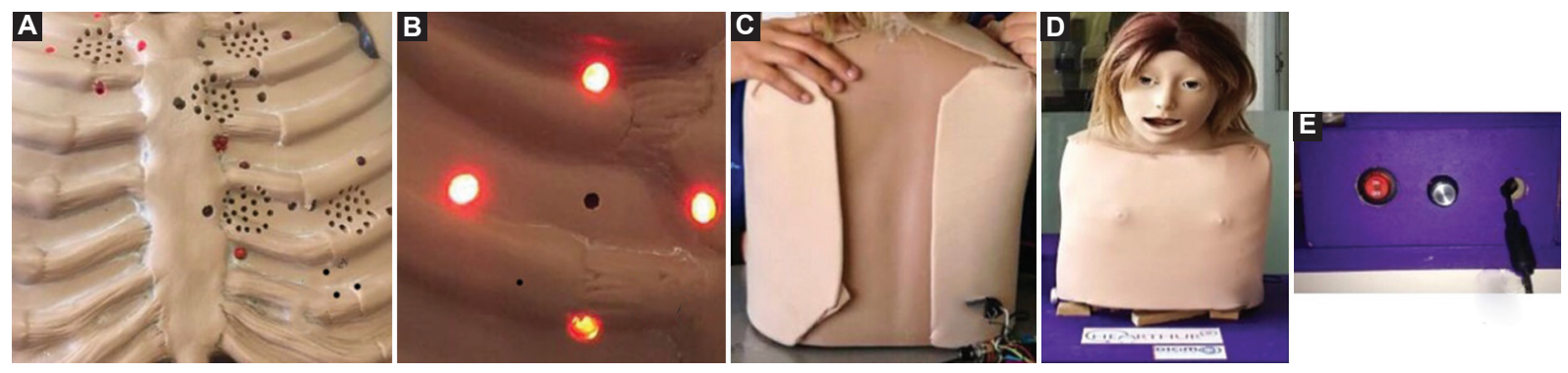

Figura 1. Proceso de elaboración del simulador Hearthur. A) Parrilla costal con las perforaciones para los focos de auscultación. B) Cuatro perforaciones para el paso del sonido donde se colocaron luces LED. C) Se unió en la parte posterior el hule espuma con una línea de pegamento velcro. D) Simulador ensamblado a la base de madera. E) Vista posterior de la caja que contiene el switch, la entrada universal y la batería.

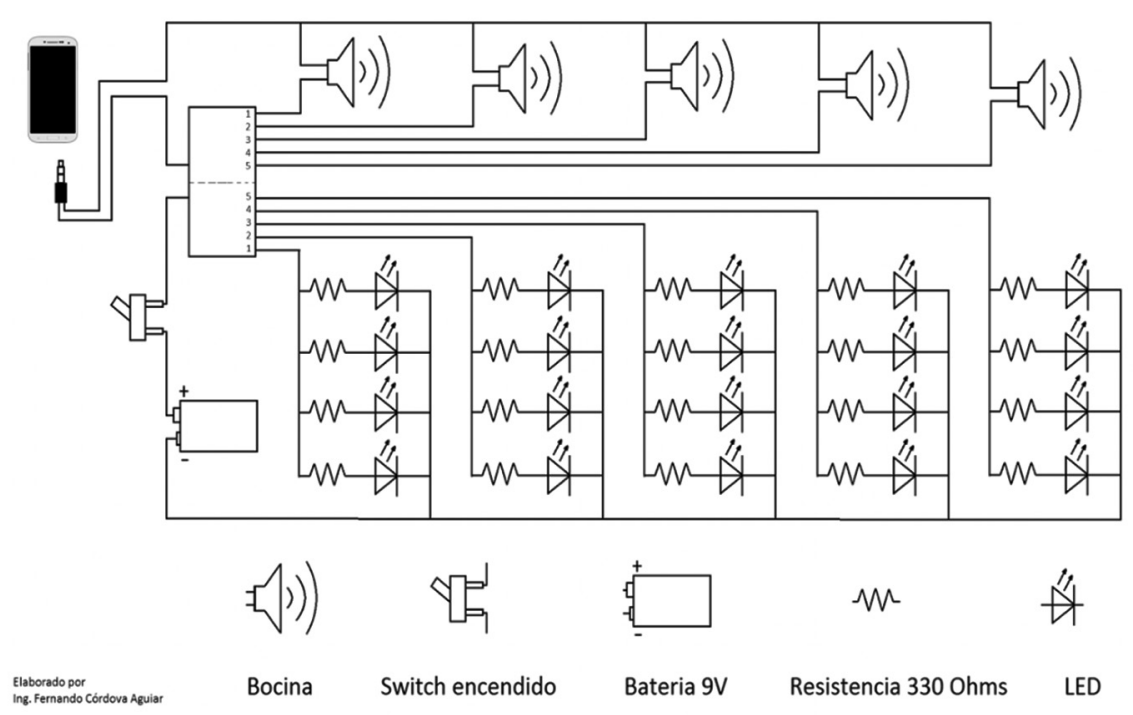

Figura 2. Circuito eléctrico y de audio. El circuito es mantenido por una batería recargable de $9 \mathrm{~V}$ y cada bocina está acompañada de cuatro luces LED. La entrada universal de audio dirige el sonido por cinco cables distintos, correspondientes a cada bocina, de tal manera que se consigue aislar sonidos de cada foco de auscultación.

joven sin antecedentes de importancia. La grabación fue guardada en formato $\mathrm{mp} 3$ de tal forma que cualquier dispositivo móvil puede reproducirla.

4. Validación del simulador Hearthur: se seleccionó una muestra total de 58 médicos (100\%) pertenecientes a la Facultad de Medicina de la Universidad Nacional Autónoma de México, de los cuales $25(43 \%)$ fueron docentes y clínicos expertos (cardiólogos) y $33(57 \%)$ fueron estudiantes de segundo año. Todos realizaron la exploración cardiaca al simulador y emitieron su opinión mediante una lista de apreciación en la que se utilizó una escala tipo Likert (Tabla 1) constituida por 14 ítems, cada uno de los cuales se calificó con una escala de 5 a 0 , donde 5 es totalmente de acuerdo; 4, de acuerdo; 3, neutral"; 2 , en desacuerdo; 1 , totalmente en desacuerdo; y 0 , no aplica. Los ítems se clasificaron en tres categorías conforme la información que indagan: si el simulador favorece el aprendizaje (ítems 1 a 4), si el aspecto morfológico y funcionamiento es real (ítems 5 a 9) y la utilidad del simulador en la enseñanza (ítems 10 a 14).

\section{Resultados}

El aspecto del simulador Hearthur se reprodujo con alto realismo, a pesar del bajo costo, así como los sonidos que escuchó el grupo de expertos para emitir su opinión respecto a su uso en la enseñanza, así como los estudiantes de medicina que lo utilizaron. 
Tabla 1. Encuesta de valoración para simulador Hearthur

Ítem

1. El simulador ayuda a reafirmar los conceptos aprendidos de la auscultación

2. El simulador ayuda a comprender las habilidades de la auscultación

3. Motiva el aprendizaje

4. El aspecto del simulador le facilitó realizar el procedimiento

5. El simulador le permitió realizar la inspección

6. El simulador le permitió realizar la palpación

7. El simulador le permitió realizar la percusión

8. El simulador le permitió realizar la auscultación

9. La calidad de los ruidos cardiacos coinciden con los de un paciente real

10. Considera útil la guía visual de luz LED para identificar cada foco cardiaco

11. Considera que es de mayor utilidad para realizar la práctica de auscultación cardiaca en primer año

12. Considera que el simulador es una herramienta atractiva para fortalecer el aprendizaje

13. Considera que este simulador permitió tener un acercamiento a la relación médico-paciente

14. Recomienda seguir utilizando el recurso

$0=$ no aplica, 1 = totalmente en desacuerdo, 2 = en desacuerdo, 3 = neutral, 4 = de acuerdo, 5 = totalmente de acuerdo.

El cuestionario indagó tres aspectos del simulador (aprendizaje, realismo y utilidad) con una apreciación de 0 a 5 , según la opinión de la muestra seleccionada.

Tabla 2. Porcentajes por categoría

\begin{tabular}{|c|c|c|c|c|c|c|}
\hline \multirow[t]{3}{*}{ Escala } & \multicolumn{6}{|c|}{ Categoría } \\
\hline & \multicolumn{2}{|c|}{ A } & \multicolumn{2}{|c|}{ B } & \multicolumn{2}{|c|}{ C } \\
\hline & Experto (\%) & Estudiante (\%) & Experto (\%) & Estudiante (\%) & Experto (\%) & Estudiante (\%) \\
\hline Totalmente de acuerdo & 70 & 82 & 61 & 68 & 85 & 74 \\
\hline De acuerdo & 22 & 17 & 20 & 25 & 11 & 21 \\
\hline Neutral & 5 & 1 & 10 & 6 & 0 & 4 \\
\hline En desacuerdo & 0 & 0 & 3 & 0 & 1 & 1 \\
\hline Totalmente en desacuerdo & 0 & 0 & 0 & 0 & 0 & 0 \\
\hline No aplica & 3 & 0 & 6 & 1 & 3 & 0 \\
\hline Total & 100 & 100 & 100 & 100 & 100 & 100 \\
\hline
\end{tabular}

$\mathrm{A}$ = favorece el aprendizaje, $\mathrm{B}=$ morfología y función real, $\mathrm{C}=$ utilidad en la enseñanza.

La mayor puntuación se ubicó en las categorías de acuerdo y totalmente de acuerdo. Ambos grupos emitieron opiniones similares.

Respecto a la escala de apreciación, los datos obtenidos fueron recopilados en una base de datos y se analizaron con el programa Excel. Se calculó el porcentaje por cada categoría de ítem, como se muestra en la Tabla 2.

En la escala "totalmente de acuerdo" y "de acuerdo", $94 \%$ opinó que el simulador motiva el aprendizaje y que el aspecto del simulador facilita realizar la exploración cardiaca, $91 \%$ resaltó que este modelo permite realizar la inspección, $94 \%$ mencionó que permite realizar la palpación, 79 \% indicó que permite realizar la percusión y $87 \%$, que permite realizar la auscultación; 92 \% mencionó que los ruidos cardiacos coinciden a los de un paciente real; $91 \%$ opinó 
que el simulador es una herramienta atractiva para fortalecer el aprendizaje de la exploración cardiaca y $98 \%$ recomendó seguir utilizándolo como un recurso para la enseñanza o el reforzamiento de esta competencia médica.

\section{Discusión}

Este estudio demuestra que el uso de un simulador realista y de bajo costo para la exploración cardiaca mejora la adquisición de habilidades para esta competencia y estimula el aprendizaje en el estudiante, además de que facilita la práctica deliberada del procedimiento antes de realizarlo en un paciente real. . $^{7-10}$

El costo elevado de los simuladores de alta fidelidad hace que la herramienta de la simulación sea poco accesible, sin embargo, la literatura médica actual recomienda la simulación clínica en los planes de estudio de medicina. ${ }^{11}$ Por ello, el diseño y la elaboración de un simulador realista y de bajo costo como Hearthur, cuyo costo total es de $\$ 1500.00$ m.n., haría posible el acceso a esta herramienta de enseñanza a un mayor número de estudiantes.

\section{Conclusiones}

Es posible la elaboración de un simulador realista y de bajo costo para la exploración cardiaca. El simulador que describimos demuestra que el aspecto de este motiva al estudiante a realizar una exploración integral del paciente al ser una herramienta atractiva, fácil y dinámica; además, favorece la adquisición de la competencia al ser una herramienta que consolida la exploración física a través de la inspección, palpación y auscultación. Además, el reforzamiento sensorial al palpar los espacios intercostales y la localización anatómica visual a través de las luces LED ayuda a que el aprendizaje sea experiencial y significativo para el estudiante.

Respecto a futuras líneas de investigación, se sugiere conseguir mayor realismo en cuanto a la forma y aspecto del simulador, la colocación de una silueta cardiaca que confiera matidez a la percusión y la recolección de diversos ruidos cardiacos compatibles con patologías (soplos, ruidos agregados, etcétera) que puedan ser almacenados en una base de datos, reproducidos desde cualquier dispositivo móvil y con menú interactivo.

\section{Agradecimientos}

Los autores agradecemos al ingeniero Fernando Córdova Aguiar, por su ayuda técnica en el diseño y elaboración del circuito eléctrico y de audio.

\section{Conflicto de intereses}

\section{Ninguno.}

\section{Fuentes de financiamiento}

La presente investigación no recibió ninguna beca específica de agencias de los sectores público, comercial, o sin ánimo de lucro.

\section{Responsabilidades éticas}

Protección de personas y animales. Los autores declaran que para esta investigación no se realizaron experimentos en seres humanos ni en animales.

Confidencialidad de los datos. Los autores declaran que en este artículo no aparecen datos de pacientes.

Derecho a la privacidad y consentimiento informado. Los autores declaran que en este artículo no aparecen datos de pacientes.

\section{Bibliografía}

1. Vukanovic-Criley JM, Criley S, Warde CM, Boker JR, Guevara-Matheus L, Churchill WH, et al. Competency in cardiac examination skills in medical students, trainees, physicians, and faculty: a multicenter study. Arch Intern Med. 2006;166:610-616.

2. Conn RD, O'Keefe JH. Cardiac physical diagnosis in the digital age: an important but increasingly neglected skill (from stethoscopes to microchips). Am J Cardiol. 2009;104:590-595.

3. McKinney J, Cook DA, Wood D, Hatala R. Simulation-based training for cardiac auscultation skills: Systematic review and meta-analysis. J Gen Intern Med. 2013;28:283-291.

4. lung $B$, Vahanian A. Epidemiology of acquired valvular heart disease. Can J Cardiol. 2014;30:962-970.

5. McGaghie WC, Issenberg SB, Cohen ER, Barsuk JH, Wayne DB. Does simulation-based medical education with deliberate practice yield better results than traditional clinical education? A meta-analytic comparative review of the evidence. Acad Med. 2011:86:706-711.

6. Bradley P. The history of simulation in medical education and possible future directions. Med Educ. 2006;40:254-262.

7. Frost DW, Cavalcanti RB, Toubassi D. Instruction using a high-fidelity cardiopulmonary simulator improves examination skills and resource allocation in family medicine trainees. Simul Healthc. 2011;6:278-283.

8. Yuan HB, Williams BA, Fang JB, Ye QH. A systematic review of selected evidence on improving knowledge and skills through high-fidelity simulation. Nurse Educ Today. 2012;32:294-298.

9. Butter J, McGaghie WC, Cohen ER, Kaye M, Wayne DB. Simulation-based mastery learning improves cardiac auscultation skills in medical students. J Gen Intern Med. 2010;25:780-785

10. Cook DA, Hamstra SJ, Brydges R, Zendejas B, Szostek JH, Wang AT, et al. Comparative effectiveness of instructional design features in simulation-based education: Systematic review and meta-analysis. Med Teach. 2013;35:e867-e898.

11. Waldrop WB, Murray DJ, Boulet JR, Kras JF. Management of anesthesia equipment failure: a simulation-based resident skill assessment. Anesth Analg. 2009;109:426-433 